\title{
Segregation of Nitrogen
} Fixation and Oxygenic Photosynthesis in the Marine Cyanobacterium Trichodesmium

\author{
Ilana Berman-Frank, ${ }^{1 *}$ Pernilla Lundgren, ${ }^{2}$ Yi-Bu Chen, ${ }^{1}$ \\ Hendrik Küpper, ${ }^{3,4,5}$ Zbigniew Kolber, $^{1}$ Birgitta Bergman, ${ }^{2}$ \\ Paul Falkowski ${ }^{1}$
}

\begin{abstract}
In the modern ocean, a significant amount of nitrogen fixation is attributed to filamentous, nonheterocystous cyanobacteria of the genus Trichodesmium. In these organisms, nitrogen fixation is confined to the photoperiod and occurs simultaneously with oxygenic photosynthesis. Nitrogenase, the enzyme responsible for biological $\mathrm{N}_{2}$ fixation, is irreversibly inhibited by oxygen in vitro. How nitrogenase is protected from damage by photosynthetically produced $\mathrm{O}_{2}$ was once an enigma. Using fast repetition rate fluorometry and fluorescence kinetic microscopy, we show that there is both temporal and spatial segregation of $\mathrm{N}_{2}$ fixation and photosynthesis within the photoperiod. Linear photosynthetic electron transport protects nitrogenase by reducing photosynthetically evolved $\mathrm{O}_{2}$ in photosystem I (PSI). We postulate that in the early evolutionary phase of oxygenic photosynthesis, nitrogenase served as an electron acceptor for anaerobic heterotrophic metabolism and that PSI was favored by selection because it provided a micro-anaerobic environment for $\mathrm{N}_{2}$ fixation in cyanobacteria.
\end{abstract}

Nitrogenase, the enzyme that catalyzes the reduction of atmospheric $\mathrm{N}_{2}$ to ammonia, is irreversibly inhibited upon exposure to molecular oxygen $(1,2)$. Cyanobacteria produce molecular oxygen via photosynthesis and have evolutionary adaptations that protect nitrogenase from oxygen; these adaptations include either a temporal separation, in which $\mathrm{N}_{2}$ fixation occurs in the dark, or a spatial segregation, in which $\mathrm{N}_{2}$ fixation is confined to a specialized cell, the heterocyst, in which only PSI remains active. The major bloomforming $\mathrm{N}_{2}$-fixing organisms (diazotrophs) in modern oceans belong to the genus Trichodesmium. This genus is characterized by nonheterocystous filaments (trichomes), which form colonies. Trichodesmium are unusual among cyanobacteria because they fix nitrogen only during the photoperiod, while simultaneously producing $\mathrm{O}_{2}(3,4)$. How nitrogenase is protected from damage by pho-

${ }^{1}$ Environmental Biophysics and Molecular Ecology Program, Institute of Marine and Coastal Sciences, Rutgers University, 71 Dudley Road, New Brunswick, NJ 08901, USA. 'Department of Botany, Stockholm University, SE-106 91 Stockholm, Sweden. ${ }^{3}$ Photosynthesis Research Center, Institute of Microbiology, Opatovický mlýn, CZ-37981 Třeboň, Czech Republic. ${ }^{4}$ Laboratory of Biomembranes, University of South Bohemia, Branišovská 31, CZ-370 05 České Budějovice, Czech Republic. ${ }^{5}$ University of Konstanz, Faculty of Sciences, Department of Biology, D-78457 Konstanz, Germany.

*To whom correspondence should be addressed. Email: irfrank@imcs.rutgers.edu tosynthetically produced $\mathrm{O}_{2}$ and how this process is regulated has been an enigma since Dugdale et al. first identified these organisms as light-dependent diazotrophs 40 years ago (3-8). In Trichodesmium, nitrogenase is localized in subsets of consecutively arranged cells in each trichome, which also contain photosynthetic components $(8,9,10)$ and comprise 15 to $20 \%$ of all cells (9-14). Here, we demonstrate that a combined temporal and spatial segregation of $\mathrm{N}_{2}$ fixation and oxygen evolution provides a window of opportunity that permits the cells to fix nitrogen for only a few hours during the photoperiod.

Using fast repetition rate fluorometry (FRRF) (15), oxygen production, and carbon and $\mathrm{N}_{2}$ fixation, we found that changes in the activity of photosystem II (PSII) reveal a temporal separation between $\mathrm{N}_{2}$ fixation and photosynthesis during the photoperiod. In the field, photosynthetic carbon fixation increased in the morning but declined at midday, when nitrogenase activity peaked (Fig. 1C) (16). High $\mathrm{N}_{2}-$ fixation rates were measured for $\sim 6$ hours surrounding the middle of the photoperiod. When $\mathrm{N}_{2}$ fixation declined, photosynthetic ${ }^{14} \mathrm{C}$ uptake increased again (Fig. 1A). This inverse relationship was even more pronounced in laboratory cultures, where an almost complete temporal separation of $\mathrm{N}_{2}$ fixation and ${ }^{14} \mathrm{C}$ fixation was also observed (Fig. 2, A and C). Moreover, the period of high $\mathrm{N}_{2}$ fixation was characterized by a decline in gross photosynthetic production, which resulted in a negative net production of oxygen (Fig. 2D). The photochemical quantum yield [variable fluorescence/maximal fluoresence $\left.\left(F_{\mathrm{v}} / F_{\mathrm{m}}\right)\right]$ of PSII varied inversely with $\mathrm{N}_{2}$ fixation in both field and cultured populations (Figs. 1 and 2). During the photoperiod, $F_{\sqrt{ }} / F_{\mathrm{m}}$ was 50 to $60 \%$ lower at the peak of $\mathrm{N}_{2}$ fixation, increasing to maximum values at the end of the photoperiod, when $\mathrm{N}_{2}$ fixation declined (Figs. $1 \mathrm{~B}$ and 2, $\mathrm{A}$ and $\mathrm{C}$ ). This characteristic diel pattern in the quantum yields was observed under both subsaturating and saturating irradiances (Figs. 1B and 2, A and C) but disappeared when $\mathrm{N}_{2}$ fixation was inhibited in cells grown with nitrate (Fig. 2B).

We used FRRF to determine temporal changes in the redox state of photosynthetic electron transport (PET) components. The rate of oxidation of the primary electron acceptor in PSII, quinone $\mathrm{A}\left(\mathrm{Q}_{\mathrm{A}}^{-}\right)$, declined from sunrise to sunset, which suggested that the electron transfer components downstream of $\mathrm{Q}_{\mathrm{A}}$ [e.g., at the plastoquinone (PQ) pool] are chemically reduced (Fig. 1, C and D) (17). The retardation of electron flow led to lower quantum yields and lower rates of photosynthetic oxygen production (Fig. 1B).

Blocking linear electron transport on the acceptor side of PSII with the inhibitors 3-3,4dichlorophenyl-1', $1^{\prime}$-dimethylurea (DCMU) and 2,5-dibromo-3-methyl-6-isopropyl- $p$ benzoquinone (DBMIB), which poise the PQ pool in either an oxidized or reduced state, respectively (18), caused an immediate decline in nitrogenase activity when applied to cultures under aerobic conditions [Web fig. 1 (19)]. Under anaerobic conditions, however, nitrogenase activity was inhibited by DBMIB, which affects both photosynthetic and respiratory pathways (20), but was not inhibited by DCMU, which inhibits only $\mathrm{Q}_{\mathrm{A}}^{-}$oxidation. These results reveal that PET is not an immediate source of electrons for nitrogenase; dark respiration, although required for $\mathrm{N}_{2}$ fixation, is inadequate as an oxygen-scavenging mechanism (21); and linear PET is required for $\mathrm{N}_{2}$ fixation under aerobic conditions [Web fig. 2 (19)]. The differential effect of DCMU under aerobic and anaerobic conditions reveals that nitrogenase is protected from oxygen by electrons supplied by PSII. This phenomenon strongly implies that oxygen is scavenged by PSI via the Mehler reaction (22) [Web fig. 2 (19)].

We used fluorescently tagged primary antibodies to nitrogenase and to D1, a core protein of the oxygen-evolving PSII reaction center (23), to examine the pattern of segregation of $\mathrm{N}_{2}$ fixation and oxygenic photosynthesis on a cellular level. D1 was present in most cells in a trichome, including those containing nitrogenase (Fig. 3C). Because the turnover of D1 is extremely rapid (24), the presence of this protein strongly implies that oxygen production and $\mathrm{N}_{2}$ fixation are not simply spatially segregated. Moreover, when 
$\mathrm{N}_{2}$ fixation is maximal, $\mathrm{H}_{2} \mathrm{O}_{2}$ (produced primarily by the reduction of $\mathrm{O}_{2}$ by the Mehler reaction) is present in most cells in the trichomes, including central zones where nitrogenase is clustered (25) (Fig. 3D).

We used a microscope equipped for twodimensional measurement of in vivo chlorophyll fluorescence kinetics (26) to further examine the spatial heterogeneity in photosynthetic activity of PSII within individual cells and between trichomes. A combination of actinic radiation, saturating flashes, and a pulsed measuring light was applied to the microscopic field, enabling high spatial resolution of measured and derived fluorescence parameters for individual cells within the trichomes. In cultures measured during the early and late stages of the photoperiod, and in nitrate-grown or stationary-phase cultures, the total fluorescence yield was homogeneous in $85 \%$ of the trichomes (Fig. 3E), although zonations were observed in $F_{\mathrm{v}} / F_{\mathrm{m}}$ (Fig. 3G). In nitrogen fixing cultures, total fluorescence was high (Fig. 3F) and the quantum yield of photochemistry in PSII was low (Fig. $3 \mathrm{H})$. The lower quantum yields were a consequence of a proportionately larger increase in the initial dark-adapted fluorescence $\left(F_{\mathrm{o}}\right)$ than in $F_{\mathrm{m}}$, implying that PSII reaction centers are reduced on the acceptor side (15). The bright inactive zones were nonuniformly distributed and were seen in whole filaments, on the tips of filaments, and in central areas of trichomes (Fig. 3F). Cells could turn photosynthetic activity (i.e., variable fluorescence) on and off within 10 to $15 \mathrm{~min}$, illustrating that in Trichodesmium, in contrast to fully evolved heterocystous cyanobacteria, all cells are photosynthetically competent, but individual cells modulate oxygen production and consumption during the photoperiod. Moreover, the increased occurrence of inactive photosynthetic zones during the hours of high $\mathrm{N}_{2}$ fixation is evidence of both temporal and spatial segregation of the two processes.

Our results (Figs. 1 to 3 ) demonstrate a combined spatial and temporal segregation of $\mathrm{N}_{2}$ fixation from photosynthesis and suggest a sequential progression of photosynthesis, respiration, and $\mathrm{N}_{2}$ fixation in Trichodesmium over a diel cycle. These pathways are entrained in a circadian pattern (27) that is ultimately controlled by the requirement for an anaerobic environment around nitrogenase (28). Light initiates photosynthesis, providing energy and reductant for carbohydrate synthesis and storage, stimulating cyclic and pseudocyclic (Mehler) electron cycling through PSI, and poising the PQ pool at reduced levels (Figs. 1A and 2, A and C) [Web fig. 2 (19)]. High respiration rates (29) early in the photoperiod (Fig. 2D) supply carbon skeletons for amino acid synthesis (the primary sink for fixed nitrogen) but simultaneously reduce the PQ pool further,
Fig 1. (A to D) Diel changes in $\mathrm{N}_{2}$ fixation, carbon uptake, and fluorescence patterns measured by a FRRF fluorometer on surface populations of Trichodesmium spp. (both colonies and free filaments) collected from the Arafura and Timor Seas from 29 October to 15 November 1999. (A) Representative pattern of $\mathrm{N}_{2}$ fixation (squares) (as measured by acetylene reduction) and acid-stable ${ }^{14} \mathrm{C}$ uptake (triangles) for T. thiebautii colonies obtained on 7 and 8 November 1999. (B to D) Data represent measurements made on sea water from $3 \mathrm{~m}$ (filtered through a $200-\mu \mathrm{m}$ net) during 7 and 8 November (solid symbols) and 11 and 12 November (open symbols), using a continuous flowthrough FRR fluorometer. Microscopic observations showed mostly free filaments and small colonies of Trichodesmium spp. in these samples. (B) Photochemical quantum yields $\left(F_{\mathrm{v}} / F_{\mathrm{m}}\right)$. Data shown are a composite of continuous FRRF measurements on 7 and 8 November (solid diamonds) and the av-

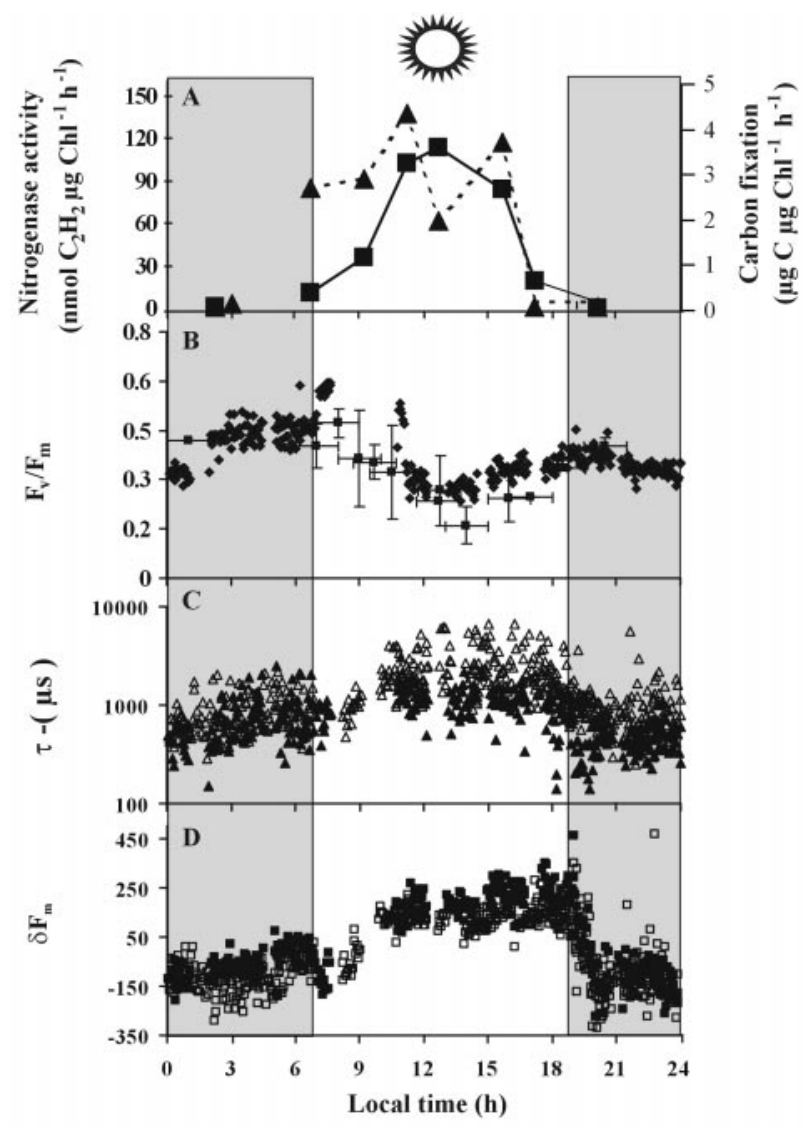
erage and standard deviations from the total samples of handpicked colonies between 29 October and 15 November 1999 (solid squares). (C) Oxidation rates of $Q_{A}^{-}(\tau]$. (D) Redox state of the PQ as estimated from $\delta F_{\mathrm{m}}\left[\delta F_{\mathrm{m}}=F_{\mathrm{m}}(\mathrm{ST}-\mathrm{MT})\right]$.
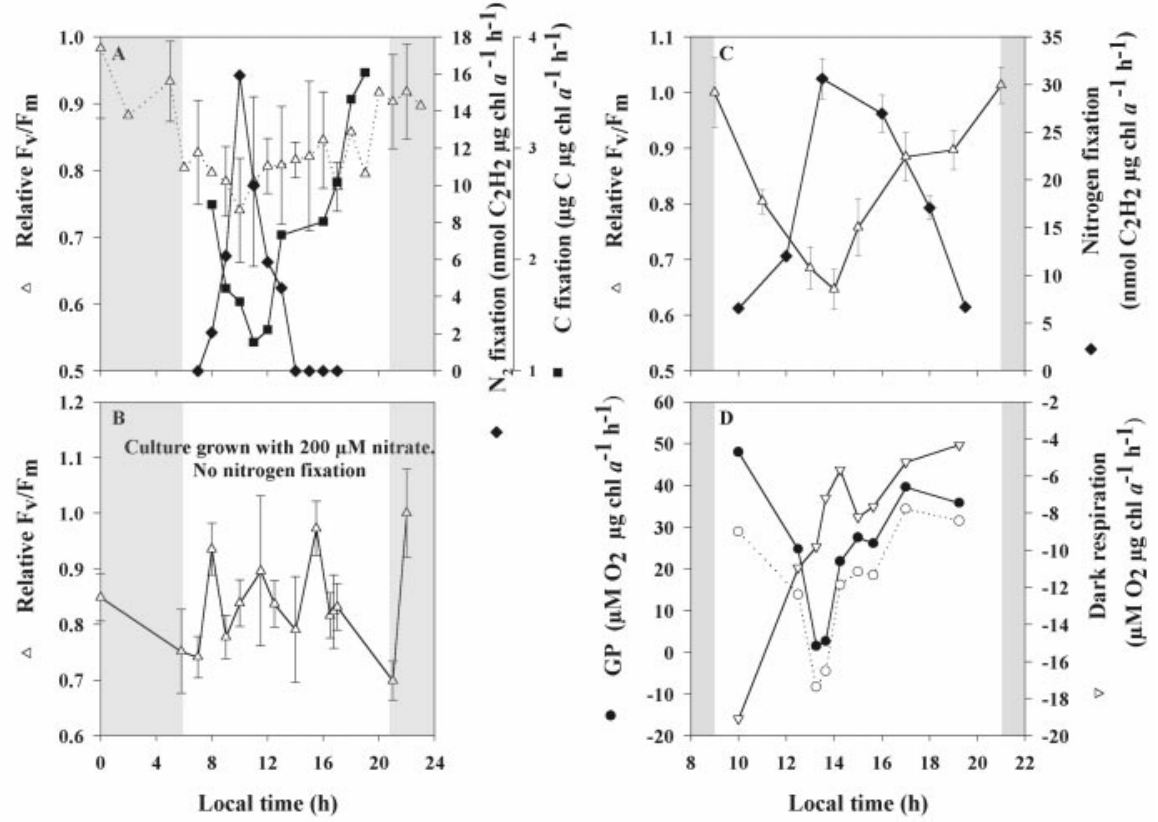

Fig. 2. Diel patterns in $\mathrm{N}_{2}$ fixation, in quantum efficiencies of PSII and in respiratory oxygen consumption and photosynthetic oxygen evolution in cultures of Trichodesmium strain IMS101. (A and B) Axenic cultures grown under a 14:10 hour light/dark cycle (L/D) under $40 \mu \mathrm{mol}$ of quanta $\mathrm{m}^{-2} \mathrm{~s}^{-1}$. (C and D) Culture grown at 12:12 L/D under $80 \mu \mathrm{mol}$ of quanta $\mathrm{m}^{-2} \mathrm{~s}^{-1}$. (A and C) Quantum yields (triangles) and acetylene reduction rates (diamonds) of culture grown under diazotrophic conditions with no inorganic nitrogen source. (B) Quantum yields (triangles) of culture grown on $200 \mu \mathrm{M} \mathrm{NO}_{3}$ exhibiting no $\mathrm{N}_{2}$ fixation as measured by the acetylene reduction method. (D) Oxygen consumption and evolution as measured on a Clark-type $\mathrm{O}_{2}$ electrode. Dark respiration (triangles), gross photosynthesis (circles), and net oxygen evolution (open circles). 
sending negative feedback to linear PET [Web fig. 2 (19)]. The reduced PQ pool leads to a down-regulation of PSII (Figs. 3, F and $\mathrm{H}$, and $1, \mathrm{C}$ and $\mathrm{D})$. However, linear electron flow to PSI is never abolished [Web fig. 2 (19)]. The down-regulation of PSII opens a window of opportunity for $\mathrm{N}_{2}$ fixation during the photoperiod, when oxygen consumption exceeds oxygen production. As the carbohydrate pool is consumed, respiratory electron flow through the PQ pool diminishes, intracellular oxygen concentrations rise, the PQ pool becomes increasingly oxidized, and net oxygenic production exceeds consumption (Figs. 1A and 2, C and D). Nitrogenase activity is lost until the following day.

The combination of spatial and temporal segregation of $\mathrm{N}_{2}$ fixation and oxygenic photosynthesis during the photoperiod appears to reflect the evolutionary history of $\mathrm{N}_{2}$ fixation in cyanobacteria. Nitrogenase is an ancient enzyme that almost certainly arose in the Archean Ocean before the oxidation of the atmosphere by oxygenic photoautotrophs $(30,31)$. We propose that under the prevailing anaerobic conditions of that period in Earth's history, $\mathrm{N}_{2}$ served
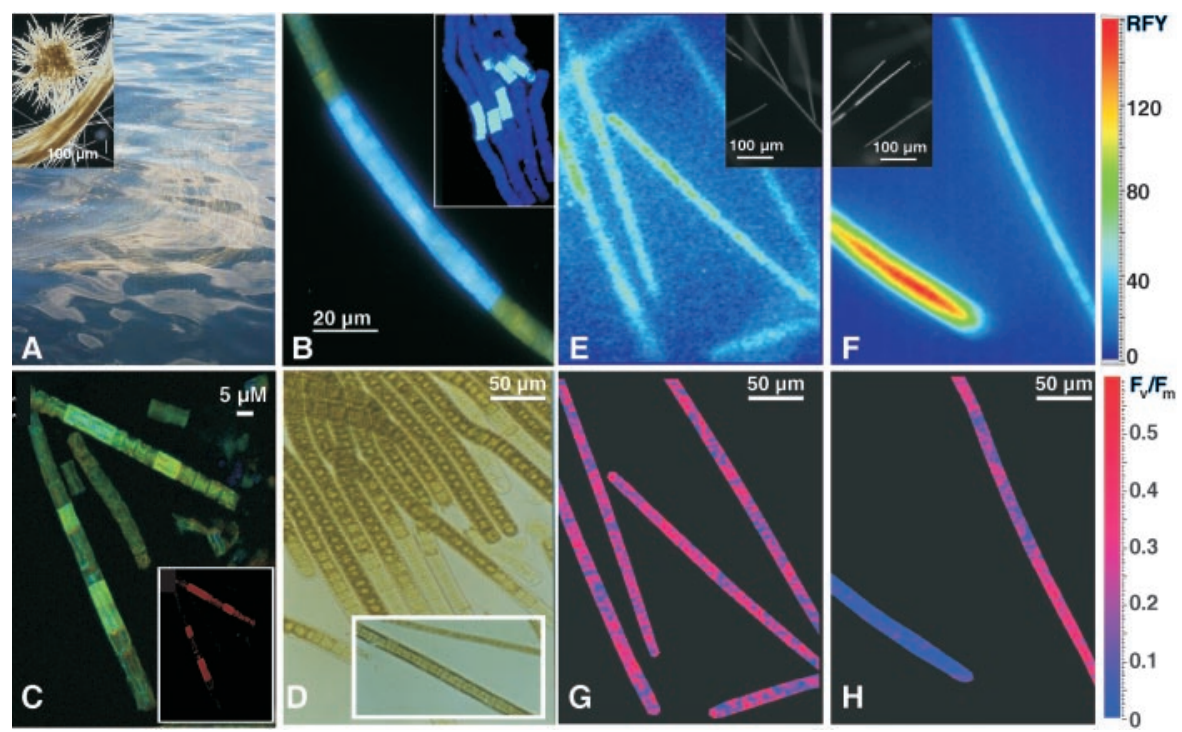

Fig 3. (A) A surface bloom of Trichodesmium spp. from the Arafura Sea. Inset: Puff and tuft formations of $T$. thiebautii colonies. (B) Nitrogenase localization in a single IMS101 trichome visualized with an epifluorescent microscope (Olympus U-MWU; BP 330 to 385, DM 400, BA 420) using a fluorescent secondary antibody Alexa-350 (Molecular Probes). Insert: Natural population of T. erythraeum probed as above. (C) Trichodesmium IMS101 probed simultaneously with fluorescently tagged primary antibodies to both D1 and nitrogenase and viewed on a confocal laser microscope (Zeiss LSM410) at 488/528 nm and 568/600 to 620 bandpass excitation/emission for D1 (green) and nitrogenase (red), respectively. The large image is the composite overlay of both channels. Insert: Nitrogenase label only. (D) Colonies of T. erythraeum, collected from surface waters of the Arafura Sea and stained with $D A B$, showing the intracellular distribution of $\mathrm{H}_{2} \mathrm{O}_{2}$ as a brown stain throughout the cells during peak hours of $N_{2}$ fixation (13:30 to 14:30). Insert: Midsection of a single trichome stained with brown deposits. (E to $\mathbf{H}$ ) Trichomes of cultured Trichodesmium IMS101 viewed with a microscope for two-dimensional measurements of in vivo chlorophyll fluorescence kinetics (45). (E and G) Trichomes from the early hours (00:00 to 04:00) of the photoperiod when $\mathrm{N}_{2}$ fixation is low. $\left(\mathrm{F}\right.$ and $\mathrm{H}$ ) Trichomes from hours of high $\mathrm{N}_{2}$ fixation ( 5 to 7 hours into the photoperiod). (E and F) Total chlorophyll fluorescence. Inserts: Trichomes photographed with a nonamplified high-resolution camera showing the pattern of normal and bright filaments (i.e., high total fluorescence to very low $F_{v} / F_{m}$ ) for the corresponding sampling times. Scale bar indicates relative fluorescence yield. $(G$ and $H)$ False color images of the two-dimensional distribution of PSIl efficiency, $F_{\mathrm{v}} / F_{\mathrm{m}}$ along the trichomes.

\section{References and Notes}

1. P. W. Ludden, G. P. Roberts, in Anoxygenic Photosynthetic Bacteria, R. E. Blankenship, M. T. Madigan, C. E. Bauer, Eds. (Kluwer Academic, Dordrecht, Netherlands, 1995), pp. 929-947.

2. In cyanobacteria, nitrogenase consists of two proteins: the iron protein and the iron-molybdenum protein. The former is an $\alpha_{2}$ dimer with a molecular weight (MW) of about 65,000 that contains a single $\mathrm{Fe}_{4} \mathrm{~S}_{4}$ cluster bound between subunits. The latter is an $\alpha_{2} \beta_{2}$ heterotetramer, of approximate MW of 250,000 with each unit containing two types of clusters, the P cluster and the FeMoco center (sometimes called the $\mathrm{M}$ cluster). The $\mathrm{P}$ cluster is an $\mathrm{Fe}_{8} \mathrm{~S}_{7}$ center that functions as a conduit for electron transfer, accepting electrons from the $\mathrm{Fe}_{4} \mathrm{~S}_{4}$ cluster of the iron protein [in conjunction with adenosine triphosphate (ATP) hydrolysis] and donating them to the FeMoco center, which is the site of $\mathrm{N}_{2}$ reduction. Whereas both the $\mathrm{Fe}_{4} \mathrm{~S}_{4}$ and $\mathrm{P}$ clusters are inactivated by $\mathrm{O}_{2}$, the $\mathrm{Fe}_{4} \mathrm{~S}_{4}$ cluster is much more susceptible and irreversibly damaged (34).

3. R. C. Dugdale et al., Deep Sea Res. 7, 297 (1961).

4. T. Saino, A. Hattori, Deep Sea Res. 25, 1259 (1978).

5. E. J. Carpenter, C. C. Price, Science 191, 1278 (1976)

6. D. G. Capone et al., Science 276, 1221 (1997)

7. B. Bergman et al., FEMS Microbiol. Rev. 19, 139 (1997).

8. H. W. Paerl, J. Phycol. 30, 790 (1994)

9. S. J. Lin et al., App. Environ. Microbiol. 64, 3052 (1998).

10. B. Bergman, in Marine Nitrogen-Fixing Cyanobacteria, L. Charpy, T. Larkum, Eds. (Marine Cyanobacteria, Bulletin Institute Oceanographic, Monaco, 1999), pp. 158-163.

11. S. Janson et al., FEMS Microbiol. Lett. 118, 9 (1994).

12. C. Fredriksson, B. Bergman, Microbiology 141, 2471 (1995).

13. Protoplasma 197, 76 (1997)

14. Primary $\mathrm{NifH}$ antibody was raised in rabbit against the Fe protein from Rhodospirillum rubrum and was visualized with an epifluorescent microscope (Olympus BX-60 ultraviolet light) [excitation filter (BP) 330 to 385 , dichroic mirror (DM) 400 , barrier filter (BA) $420 \mathrm{~nm}$ ] by using a secondary antibody coupled to Alexa-350 (Molecular Probes).

15. FRRF measures fluorescence transients induced by a series of subsaturating excitation pulses (35). $F_{\text {o }}$ is measured when $\mathrm{Q}_{\mathrm{A}}$ is fully oxidized; $F_{\mathrm{m}}$ occurs when $\mathrm{Q}_{\mathrm{A}}$ is fully reduced. $F_{\mathrm{v}}=\left(F_{\mathrm{m}}-F_{\mathrm{o}}\right)$, and the photochemical quantum yield of PSII $\left(F_{\mathrm{v}} / F_{\mathrm{m}}\right)(36)$ correlate well with independent measurements of photosynthetic competence such as yield of oxygen evolution (37).

16. Field populations of Trichodesmium erythreum and $T$. thiebautii were collected and analyzed in the Arafura and Timor Seas, north of Australia, between 21 October and 16 November 1999. Colonies and filaments were obtained with net tows at varying depths, handpicked, and resuspended in filtered sea water. For continuous FRRF measurements sea water was pumped from a 3-m depth and filtered through 200 $\mu \mathrm{m}$ mesh before entering the FRRF at a flow of 240 $\mathrm{ml} / \mathrm{min}$ Trichodesmium species composition was monitored microscopically.

17. $\delta F_{\mathrm{m}}=F_{\mathrm{m}}(\mathrm{ST}-\mathrm{MT})$, where $\mathrm{ST}$ and $\mathrm{MT}$ are single- and multiple-turnover flashes, respectively (15).

18. DCMU binds competitively with the secondary quinone acceptor $\left(Q_{B}\right)$ and prevents the reduction of the $P Q$. DBMIB prevents the oxidation of the $P Q$ by blocking the plastoquinol binding site of cytochrome $\left.b_{6} f(38).\right]$.

19. Supplementary Web material is available on Science Online at www.sciencemag.org/cgi/content/full/294/ 5546/1534/DC1.

20. In cyanobacteria, $\mathrm{NAD}(\mathrm{P}) \mathrm{H}$ carries electrons produced by respiratory oxidation into the photosynthetic electron transport chain via the thylakoidbound dehydrogenase(s), which subsequently reduces the PQ and the cytochrome $b_{6} f$ complex, which is a plastoquinol oxidoreductase and lies downstream of the PQ just before reaching PSI. Thus, DBMIB inhibits both photosynthetic and respiratory electron flow downstream of cytochrome $b_{6} f(39)$. 
21. Local zones of higher respiratory activity may be important, however, as evidenced by higher levels of the respiratory enzyme cytochrome oxidase in the subsets of cells with nitrogenase than in those without (40). Deployment of oxygen and reactive oxygen species detoxification systems (such as Mehler, thioredoxin peroxidases, and catalase) also aid in providing a microanaerobic environment around cells fixing nitrogen. Colony formation may further reduce ambient oxygen concentrations $(5,8)$, enabling the higher $\mathrm{N}_{2}$ fixation rates (per unit of chlorophyll a) observed in colonies as compared to single trichomes (41).

22. In mature heterocysts, PSI is the only active photosynthetic reaction center and is important in providing the extra ATP for $\mathrm{N}_{2}$ fixation through cyclic electron transport. In Trichodesmium, high Mehler activity has also previously been invoked in supplying ATP $(42,43)$.

23. Antibodies to D1 fragments and to dinitrogenase reductase raised in rabbits were conjugated to fluorescent probes Alexa 488 and Alexa 568, respectively (Molecular Probes) and labeled sequentially (nitrogenase followed by D1) in cells fixed in $100 \%$ ethanol and permeabilized with $0.5 \%$ dimethyl sulfoxide in phosphate buffer. Samples were viewed on a confocal laser microscope (Zeiss LSM410) at $488 / 528 \mathrm{~nm}$ and $568 / 600$ to $620 \mathrm{~nm}$ bandpass excitation/emission for the D1 and nitrogenase, respectively. The results obtained for cultures grown in several conditions and at several points during the diel cycle show that D1 occurs in most cells in a trichome and co-occurs in the same cells as nitrogenase.

24. Z. Krupa, G. Öquist, P. Gustafsson, Plant Physiol. 93 1 (1990).

25. Trichodesmium colonies were stained with 3,3-diaminobenzidine (DAB), which polymerizes, in the presence of peroxidases, with intracellular $\mathrm{H}_{2} \mathrm{O}_{2}$ produced by reduction of oxygen in PSI, to form brown deposits (44). The final concentration of DAB used was $1 \mathrm{mg} / \mathrm{ml}$. No external peroxidases were added, indicating the presence of active peroxidases involved in the antioxidative pathways [Web fig. 2 (19)]. Negative controls of dark-incubated Trichodesmium trichomes showed low DAB staining throughout the trichomes.

26. Trichomes were filtered, embedded in $1 \%$ agarose (melting point $25^{\circ} \mathrm{C}$ ) in sea water, and placed in a cellophane-sealed thermostated chamber pumped

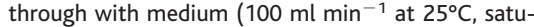
rated with air). To reduce artifacts caused by handling, fresh samples were prepared for each time point. Samples were viewed with a microscope for two-dimensional measurements of in vivo chlorophyll fluorescence kinetics (45). Measurements were done with 30- $\mu$ s flashes of nonactinic measuring light, $1000 \mu \mathrm{mol}$ of quanta $\mathrm{m}^{-2} \mathrm{~s}^{-1}$ of actinic light, and $10,000 \mu \mathrm{mol}$ of quanta $\mathrm{m}^{-2} \mathrm{~s}^{-1}$ saturating multiturnover flashes. Fluorescence kinetics were measured simultaneously on $300 \times 400$ pixels.

27. A circadian pattern temporally separates the abundance of mRNA for nifH (nitrogenase), psbA (encoding for PSII) and psaB (encoding for PSI) in Trichodesmium strain IMS101 $(46,47)$.

28. In Trichodesmium, high external concentrations of molecular oxygen affect nitrogenase activity within $\sim 15$ min (48), whereas Western and Northern blots of nitrogenase and nifH (49) revealed that the enzyme and transcript levels are not much affected 2 hours after addition of DCMU and DBMIB, indicating that the loss of activity is not due to the loss of the enzyme but rather to a posttranslational inactivation of the enzyme by oxygen.

29. In most cyanobacteria, dark respiration rates are generally $<10 \%$ of the gross oxygen evolution rates (50). In Trichodesmium, dark respiration ranged from 13 to $46 \%$ of the maximum gross oxygen evolution rate, with a mean of $23 \%$ and consisting, in the dark, of approximately $30 \%$ of the absolute magnitude of maximal gross photosynthesis. Moreover, at low light intensities (typical of those found for depth-adapted populations or cultured populations), more oxygen was consumed than evolved $(51,52)$

30. Phylogenetic analyses suggest a single ancestral ori- gin for the catalytic subunits of the enzyme complex responsible, namely nitrogenase (53).

31. P. G. Falkowski, Nature 387, 272 (1997).

32. J. P. Zehr, M. T. Mellon, S. Zani, Appl. Environ. Microbiol. 64, 3444 (1998)

33. C. P. Wolk, A. Ernst, J. Elhai, in The Molecular Biology of Cyanobacteria, D. E. Bryant, Ed. (Kluwer Academic Dordrecht, Netherlands, 1994), pp. 769-823.

34. B. K. Burgess, D. J. Lowe, Chem. Rev. 96, 2983 (1996)

35. Z. Kolber, O. Prašil, P. G. Falkowski, Biochim. Biophys. Acta 1367, 88 (1998)

36. G. H. Krause, E. Weis, Annu. Rev. Plant Physiol. Plant Mol. Biol. 42, 313 (1991)

37. D. Campbell et al., Microbiol. Mol. Biol. Rev. 62, 667 (1998).

38. P. G. Falkowski, J. A. Raven, Aquatic Photosynthesis (Blackwell Science, Malden, MA, ed. 1, 1997).

39. M. Hirano, K. Satoh, S. Katoh, Photosyn. Res. 1, 149 (1980)

40. B. Bergman et al., Appl. Environ. Microbiol. 59, 3239 (1993).

41. R. M. Letelier, D. M. Karl, Aquatic Microbiol Ecol. 15, 265 (1998).

42. T. M. Kana, in Marine Pelagic Cyanobacteria: Trichodesmium and Other Diazotrophs, E. J. Carpenter, Ed. (Kluwer Academic, Dordrecht, 1992), pp. 29-41. 43. Limnol. Oceanogr. 38, 18 (1993).

44. H. Thordal-Christensen et al., Plant J. 11, 1187 (1997).

45. H. Küpper, I. Šetlik, M. Trtilek, L. Nedbal, Photosynthetica 38, 553 (2000).

46. Y. B. Chen et al., J. Bacteriol. 180, 3598 (1998).

47. , Plant Mol. Biol. 41, 89 (1999).

48. P. Lundgren, Y. Gerchman, unpublished data.

49. I. Berman-Frank et al., unpublished data.

50. J. C. P. Matthijs, H. J. Lubberdin, in Biochemistry of the
Algae and Cyanobacteria, Proc. Phytochem. Soc. Eur. (Clarendon, 1988) pp. 131-145.

51. T. Roenneberg, E. J. Carpenter, Mar. Biol. 117, 693 , (1993).

52. E. J. Carpenter, T. Roenneberg, Mar. Ecol. Prog. Ser 118, 267 (1995).

53. J. P. Zehr, E. J. Carpenter, T. A. Villareal, Trends Microbiol. 8, 68 (2000).

54. We thank D. Capone, E. Carpenter, and the captain and crew of the R/V Ewing for enabling the field study; R. Dotson for setting up the continuous flow to the FRR fluorometer; $K$. Bateman (Stockholm University) and K. Wyman (Rutgers University) for technical assistance; J. Waterbury (Woods Hole Oceanography Institute) for providing axenic cultures, lab space, invaluable help, and ideas; P. Ludden (University of Wisconsin), S. Nordlund (Stockholm University), and A. Matoo (U.S. Department of Agriculture) for their gift of antibodies; O. Prašil, I. Šetlik, and the Microbiological Institute, Trebon, for hosting I.B.-F. [Czech (CZ)-NSF grant ME379 and Ministry of Education of the Czech Republic grant MSM 12310001] and providing access to the kinetic microscope, which was built in cooperation with Photon Systems Instruments, Czech Republic. Supported through grants to P.F. from the U.S. Department of Energy Office of Science program for Research on Ocean Carbon Sequestration, the Center for Bioinorganic Chemistry (Princeton University), NASA Earth System Science Program; to B.B. from the Swedish Foundation for International Cooperation in Research and Higher Education and the Swedish Natural Science Research Council (SIDA/SAREC); and to H. K. from Studienstiftung des Deutschen Volkes. 